\title{
Dificuldades Ortográficas na Escrita no Ensino Fundamental
}

\author{
Maria Cristina Rodrigues Azevedo Joly \\ Débora Pereira de Barros \\ Universidade São Francisco \\ Janete Aparecida da Silva Marini \\ Centro Universitário Padre Anchieta
}

\begin{abstract}
RESUMO
O presente estudo teve por objetivo identificar o desempenho em escrita de crianças que frequentavam regularmente o ensino fundamental. Foi aplicada uma prova de escrita em 546 crianças, com faixa etária entre 7 e 13 anos, de ambos os sexos, que frequentavam $2^{\mathrm{a}}$ e $3^{\mathrm{a}}$ séries de escolas públicas e privadas do estado de São Paulo. Os resultados revelaram que os participantes da $3^{\text {a }}$ série apresentaram um desempenho melhor do que os da $2^{\mathrm{a}}$ série, apesar de apresentarem pontuação máxima e mínima igual. A categoria na qual foi verificada maior dificuldade foi a das palavras com sílabas compostas, enquanto àquelas com encontros consonantais e dígrafos foram as que os estudantes mais acertaram. Ao lado disso, os estudantes da escola particular revelaram maior competência em escrita ortográfica do que os que frequentavam as escolas públicas. Quanto ao gênero, apenas não houve diferença significativa de desempenho entre meninas e meninos para os itens com sílaba composta e complexa, nos demais itens as meninas apresentaram os melhores resultados.
\end{abstract}

Palavras-chave: escrita; rendimento acadêmico; avaliação.

\section{ABSTRACT \\ Ortographic Difficulties in Writing at a Basic Grade Level}

This study aimed to identify the performance in children's writing at a basic grade level. A written test was applied to 546 grade 2 or 3 boys and girls (aged 7 to 13) in public and private schools from the State of São Paulo. The results showed that the grade 3 participants presented a better performance than the grade 2 participants even though both groups have achieved the same maximum and minimum scores. It was confirmed that participants had more difficulty with words containing compound syllables, while words with aggregated consonants and digraphs were associated with the highest rate of correct answers. Furthermore, students from private school revealed more competence in orthographic writing than those coming from public schools. As to gender differences, there were no significant differences in performance for compound and complex syllables items though for other words, girls had better scores.

Keywords: writing; academic achievement; evaluation.

O sucesso ou fracasso escolar é um dentre os vários aspectos que influenciam o processo de desenvolvimento de crianças e jovens. Assim, dada à relevância do desempenho acadêmico, principalmente no início da escolarização quando da aquisição da leitura e escrita, faz-se necessário compreender os processos de aprendizagem, bem como, as dificuldades para aprender. Ao lado disso, há que se diferenciarem problemas de distúrbios de aprendizagem (Center for Mental Health in Schools, 2002).
As dificuldades escolares ou problemas de aprendizagem são obstáculos ou barreiras encontradas por alunos durante o período de escolarização e se manifestam basicamente por meio de atrasos e dificuldades em leitura, escrita e cálculos, em crianças de qualquer idade com inteligência potencialmente normal ou superior e sem deficiências visuais, auditivas, motoras ou desvantagens culturais. Podem ser prevenidas por estarem relacionadas ao processo de ensino e surgirem, na maioria das vezes, em situações formais de 
aprendizagem na escola. Já os distúrbios de aprendizagem referem-se às disfunções neurológicas ou físicas que implicam em defasagens perceptuais e/ou cognitivas. Diferem em grau de comprometimento dos problemas de aprendizagem, bem como, não são passíveis de prevenção (Center for Mental Health in Schools, 2002; Flanagam, Ortiz, Alfonso \& Mascolo, 2002).

Na perspectiva de Sánchez (1998), as dificuldades de aprendizagem são uma das explicações para o baixo rendimento escolar. É frequente haver alunos que experimentam algum fracasso na aprendizagem que se constata pelo atraso de um ou mais anos em relação à idade/série frequentada ou pelo abandono da escola. Em geral, esses pertencem a grupos econômicos e culturalmente desfavorecidos. Ao lado disso, Sisto (2002) aponta que as dificuldades de aprendizagem podem ser consideradas como um fenômeno, independente do ensino formal e do nível cognitivo, pois podem ocorrer ao longo da vida das pessoas e ser situacional.

Cabe considerar no contexto de aprendizagem formal que as crianças ao iniciarem a aquisição da linguagem escrita já tenham algum conhecimento de como as palavras são pronunciadas, tanto quanto do próprio alfabeto, de modo que sua tarefa seja aprender a escrever a forma impressa da língua (Snow, 2002). Segundo revisão da literatura feita por Guimarães (2003), há um grande número de estudos indicando que o desempenho das crianças pré-escolares em determinadas tarefas de consciência fonológica está diretamente relacionado ao sucesso na aquisição da leitura e da escrita. Nesse sentido, França, Wolf, Mooje e Rotta (2004) verificaram que as crianças cuja aquisição fonológica era incompleta aos 6 anos de idade demonstraram tendência a apresentarem dificuldades em leitura e escrita.

Faz-se necessário esclarecer que o desempenho em escrita nos primeiros anos de escolarização é o foco de investigação do presente estudo. Isto posto, assume-se a perspectiva apresentada por Sisto (2001) que considera estarem as dificuldades de aprendizagem na escrita quase que exclusivamente relacionadas às operações implícitas no reconhecimento dos sons que fazem parte de uma palavra, sua transformação em signos e a escrita propriamente dita desses símbolos.

É relevante considerar que o desenvolvimento das Tecnologias da Informação e Comunicação nos últimos dez anos tem promovido mudanças nos padrões de comunicação escrita nos diferentes contextos (aca- dêmico, familiar e do trabalho, por exemplo) no que se referem aos seus meios, propósitos e práticas (De Voss, Cushman \& Grabill, 2005). Acrescente-se a isto, a constatação feita na literatura específica acerca do tema de que escrever é um complexo e difícil desafio enfrentado pelo aluno em sua vida acadêmica, mesmo se tratando de uma habilidade fundamental e necessária para se expressar ou comunicar o conhecimento (Joly, 2004), requerendo pois, investigação.

\section{Pesquisas sobre escrita}

Pesquisas acerca das características do processo de aquisição e utilização da escrita têm sido realizadas por diversas áreas do conhecimento a fim de melhor caracterizarem-se as variáveis envolvidas como analisaram Capellini, Tonelotto e Ciasca (2004). Jaeger, Schossler e Wainer (1998), visando analisar a aquisição da escrita, realizaram um estudo a fim de verificar se havia diferenças entre crianças e adultos nesse processo. Participaram 32 estudantes, divididos em dois grupos - um com 16 crianças e outro com 16 adultos - sendo que todos se encontravam no segundo ano de alfabetização. Foi aplicado um ditado de 10 vocábulos como recurso avaliativo. O desempenho dos participantes foi analisado considerando o conteúdo semântico e ortográfico das palavras ditadas. Os dados revelaram que as crianças tiveram desempenho superior ao dos adultos em palavras simples. Verificou-se também, que os adultos apresentaram índice significativamente maior de erros do que as crianças. No geral, se constatou que os adultos apresentaram maior dificuldade na realização da tarefa e diferenças entre o processo de aquisição da linguagem escrita entre crianças e adultos.

Para verificar a relação entre a ortografia das palavras e a produção de textos, Berninger, Vaughan, Abbott, Brooks, Reed e Graham (1998) avaliaram protocolos de instrução designados para ensinar, tanto princípios básicos de escrita quanto de composição avançados. Participaram 128 crianças com idade média de 7,95 anos, sendo 76 meninos e 52 meninas, que frequentavam a $2^{\mathrm{a}}$ série do ensino básico em escolas públicas que foram identificadas pelos professores como tendo dificuldades com a escrita. O protocolo de instrução avaliado foi composto por 20 sessões de 24 minutos. Cada sessão foi organizada em três momentos: 5 minutos de instrução direcionada ao treinamento dos princípios do alfabeto, 7 minutos de vocabulário oral e escrito e 8 minutos para compor uma redação e lê-la em voz alta. Também foram utilizados 
jogos com letras do alfabeto para o ensino das sílabas e dicionários. Foram feitas avaliações pré e pós-programa para os participantes e análise de protocolos de auto-avaliação preenchidos pelos tutores que desenvolveram o programa como medidas de avaliação. Os resultados revelaram que mais de uma forma de desenvolver conexões entre sons e palavras é eficiente no ensino da ortografia, mas isso apenas após treinamento adequado nos princípios do alfabeto. Verificou-se ainda, que a rima foi mais eficaz no processo de transferência entre os princípios do alfabeto e a formação de palavras quando da combinação de palavras inteiras. As unidades funcionais de ortografia com duas letras ou mais também se revelaram importantes no treinamento ortográfico, pois facilitaram a composição e reconhecimento de palavras.

Frente à necessidade de caracterizar-se a produção contemporânea em pesquisa na área da escrita, Juzwik, Curcic, Wolbers, Dimling e Shankland (2006) realizaram uma meta-análise sobre temas e faixa etária, bem como, as relações entre ambas, além das metodologias utilizadas no período de 1999 a 2004. Para tanto analisaram 1.502 artigos de pesquisa de periódicos científicos provenientes do ERIC, PsychINFO e Linguistics and Language Behavior Abstracts, cujas bases são consideradas de referência para a Psicologia. Contexto social e estilos de escrita, competência linguística para duas ou mais línguas e escrita e métodos de ensino da escrita foram os problemas mais estudados. Temáticas relativas à escrita e tecnologias, avaliação da escrita e interação entre a escrita e modalidades de leitura e procedimento de cálculos foram investigadas com menor frequência. Se por um lado a maioria das pesquisas foi realizada com estudantes universitários e adultos, por outro as relativas a métodos de instrução destinaram-se aos pré-escolares e estudantes do ensino básico. O método qualitativo foi a opção preferencial para análise.

Outros estudos com estudantes em fase de aquisição da escrita e /ou no início da escolarização foram realizados no exterior (Goswami \& Bryant, 1997; Juel, Griffith \& Gough, 1986; Stanovich, Cunningham \& Cramer, 1984; Treiman \& Baron, 1983; Yopp, 1988, por exemplo) acerca das habilidades cognitivas como consciência fonológica, consciência sintática, memória, sequenciamento dentre outras, que podem ser preditivas do desempenho em escrita. No Brasil cabe destacar as investigações de Cardoso-Martins (1995), Maluf e Barrera (1997) e Capovilla (1999) como referências na área.
Mais recentemente, estudos de Guimarães (2003) sobre habilidades metalinguísticas, Capovilla, Gutschow e Capovilla (2004) sobre habilidades cognitivas, Cardoso-Martins e Batista (2005), sobre fonologia das letras do alfabeto e desempenho em escrita, Bernardino Junior, Freitas, Souza, Maranhe e Bandine (2006) acerca do desenvolvimento da habilidade em consciência fonológica e o efeito sobre aquisição de leitura e escrita, dentre outros foram realizados. Tais investigações têm comprovado a hipótese da consciência fonológica como preditiva de desempenho em escrita e destacam a necessidade de instrumentos de avaliação da escrita. Essa necessidade se assenta na possibilidade de utilizando-se instrumentos válidos e fidedignos poder-se ter uma identificação de crianças com potencial risco de apresentarem futuras dificuldades na escrita, o que possibilitaria intervenção precoce eficaz.

Em geral, as investigações acerca da avaliação do desempenho em escrita, de acordo com estudos brasileiros descritos na literatura científica dos últimos dez anos têm se baseado em provas de ditado (França, Wolf, Mooje \& Rotta, 2004; Meirelles \& Correa, 2005; Pinheiro \& Rothe-Neves, 2001; Zorzi, 1998) as quais não possuem características psicométricas declaradas e são usadas apenas para fins das pesquisas em que estão inseridas. Constataram que o desempenho dos estudantes que frequentam séries mais avançadas é melhor que os de séries iniciais do ensino fundamental. Além disso, a revisão da literatura feita por Meirelles e Correa (2005) revela que há diferentes níveis de complexidades nos contextos ortográficos sendo, pois, tais diferenças determinantes para a aquisição da escrita.

A fim de buscar critérios para diferenciar níveis de dificuldade de aprendizagem na aquisição da escrita, em especial às dificuldades de representação de fonemas, Sisto (2001a) construiu a Escala de Avaliação de Dificuldades na Aprendizagem da Escrita (ADAPE). Essa escala conta com vários estudos que lhe conferiram características psicométricas, viabilizando seu uso como recurso avaliativo. Identificaram-se diferenças significativas entre a pontuação total do ADAPE e as variáveis idade, gênero e natureza jurídica da escola, pública ou particular (Bartholomeu, Sisto \& Rueda, 2005; Cunha, Sisto \& Machado, 2006; Nunes, 2002; Pacheco \& Sisto, 2005; Sisto, 2001; Sisto \& Fernandes, 2004; Suehiro, 2006; Suehiro \& Santos, 2005).

O fato de apenas $43 \%$ dos ingressantes no sistema educacional brasileiro terminarem o ensino fundamental, acrescido da constatação de que $59 \%$ dos alu- 
nos de $4^{\mathrm{a}}$ série do ensino fundamental não dominam a linguagem segundo os resultados do Sistema Nacional de Avaliação da Educação Básica (SAEB) de 2003 (INEP, 2006) foram elementos determinantes para a realização do presente estudo. O objetivo da investigação foi verificar o padrão de escrita em estudantes em processo de aquisição desta habilidade que frequentavam escola pública e particular, bem como, analisar se há influência de gênero, idade e série frequentada sobre o desempenho em escrita.

\section{MÉTODO}

\section{Participantes}

Participaram da pesquisa 546 crianças que frequentavam regularmente a $2^{\mathrm{a}}$ e $3^{\mathrm{a}}$ série do Ensino Fundamental, sendo 278 do gênero feminino, correspondendo a $50,9 \%$ dos participantes. A faixa etária variou entre 7 e 13 anos de idade, com média de 8,65 anos e desvio padrão de 1,06. A amostra foi escolhida por conveniência e a pesquisa realizada em quatro escolas situadas em três cidades do interior de São Paulo, distribuídas conforme Tabela 1.

TABELA 1

Descrição Percentual dos Participantes por Escola

\begin{tabular}{cccc}
\hline Escola & $\mathbf{N}$ & Porcentagem & Tipo \\
\hline 1 & 159 & 30,4 & municipal \\
2 & 35 & 6,7 & particular \\
3 & 147 & 28,1 & estadual \\
4 & 182 & 34,8 & SESI \\
\hline Total & 523 & 100,0 & \\
\hline
\end{tabular}

\section{Instrumento}

\section{Avaliação de Dificuldades na Aprendizagem da Escrita - ADAPE (Sisto, 2001).}

É constituída por um texto com 114 palavras, sendo que 60 delas apresentam algum tipo de dificuldade. Essas estão classificadas como encontro consonantal (lt, mb, mp, nc, nç, nd, ng, ns, nt, rc, rd, rs, rt, sp, st), dígrafo (ch, lh, nh, qu, rr, ss), sílaba composta (br, cr, dr, gr, tr) e sílaba complexa (ão, ça, ce, ci, ge, sa, sá, se, sé, si, so), cujas características psicométricas foram consideradas suficientes para a avaliação da escrita. As outras 54 palavras do instrumento não têm dificuldade ortográfica. Cada uma dessas palavras é considerada um item de avaliação. A tarefa da criança é escrever as palavras ditadas por um aplicador. Cada palavra é ditada uma vez e repetida também uma vez. São contados os erros de ortografia, ausência de palavra, acentuação errada, uso indevido de maiúsculo e minúsculo. A pontuação máxima é de 114 pontos e corresponde à soma dos erros cometidos.

Os critérios de classificação de Dificuldade de Aprendizagem (DA) na Escrita, propostos por Sisto
(2001) diferem por série. Para os alunos da segunda série, até 20 erros é considerado sem indícios de DA, de 50 a 79 erros apresentam DA leve e a partir de 80 erros há uma DA média; para as terceiras séries até 10 erros é tido como sem indícios de DA, de 11 a 19 erros com DA leve, de 20 a 49 erros com DA média e 50 erros ou mais com DA acentuada.

\section{Procedimento}

Os procedimentos éticos para a realização da pesquisa foram realizados para que se procedesse à coleta de dados por sala e série em cada uma das quatro escolas participantes. As escolas foram previamente contatadas pelas pesquisadoras e o projeto de investigação apresentado à direção e coordenação pedagógica. Após aprovação da escola, foram agendadas as aplicações, por série. A aplicação da prova foi realizada em todas as classes por um mesmo avaliador previamente treinado que seguiu as orientações do teste para sua realização. A aplicação durou 30 minutos, em média. Após a análise dos dados e elaboração do relatório de pesquisa, realizou-se uma reunião com direção, coordenação pedagógica e professores em cada escola participante da investigação para apresentação 
dos resultados gerais por série. Tal devolutiva possibilitou uma discussão sobre procedimentos de intervenção apropriados às necessidades dos estudantes.
Os resultados revelaram que os participantes apresentaram um desempenho médio de 77,51 pontos com desvio padrão de 28,76 na avaliação em escrita. Os resultados por série estão na Tabela 2 .

\section{RESULTADOS E DISCUSSÃO}

TABELA 2

Desempenho no ADAPE por Série

\begin{tabular}{ccccccc}
\hline Série & $\mathbf{N}$ & Desempenho & $\begin{array}{c}\text { Pontuação } \\
\text { mínima }\end{array}$ & $\begin{array}{c}\text { Pontuação } \\
\text { máxima }\end{array}$ & Média & Desvio padrão \\
\hline \multirow{2}{*}{$2^{\text {a }}$} & 327 & acerto & 0 & 112 & 70,78 & 29,74 \\
& & erro & 2 & 114 & 43,48 & 29,98 \\
\hline \multirow{2}{*}{$3^{\text {a }}$} & 220 & acerto & 0 & 112 & 87,47 & 24,18 \\
& & erro & 2 & 114 & 26,53 & 24,18 \\
\hline
\end{tabular}

Verifica-se na Tabela 2 que a média de acertos dos alunos na $3^{\mathrm{a}}$ série foi maior que na $2^{\mathrm{a}}$ série, apesar de apresentarem pontuação máxima e mínima igual. Comparando o presente estudo com o de Sisto (2001), verificou-se que em ambos a média de acerto das crianças de $3^{\mathrm{a}}$ série foi melhor do que as das crianças de $2^{\mathrm{a}}$ série, sendo que apenas duas crianças de cada série $\left(2^{\mathrm{a}}\right.$ e $3^{\mathrm{a}}$ série), erraram por completo o ADAPE.

\begin{tabular}{cccc}
\multicolumn{3}{l}{$\begin{array}{l}\text { TABELA 3 } \\
\text { Classificação das Dificuldades de }\end{array}$} \\
\hline \multirow{2}{*}{ Série } & $\mathbf{N}$ & $\%$ & Classificação \\
\hline \multirow{2}{*}{$2^{\mathrm{a}}$} & 81 & 24,3 & Sem DA \\
& 57 & 17,1 & DA leve \\
& 52 & 15,6 & DA média \\
\hline \multirow{2}{*}{$3^{\mathrm{a}}$} & 61 & 27,9 & Sem DA \\
& 58 & 26,5 & DA leve \\
& 71 & 32,4 & DA média \\
& 29 & 13,2 & DA acentuada \\
\hline
\end{tabular}

A maioria dos alunos da $2^{\mathrm{a}}$ série está em processo de aquisição da escrita (43\%) e apenas $17,1 \%$ apresentaram um pouco de dificuldade, como constatado no estudo de Bartholomeu, Sisto e Rueda (2005). Na $3^{\mathrm{a}}$ série $72,1 \%$ dos participantes apresentaram Dificuldade de Aprendizagem, sendo que 13,2\% com DA acentuada. Estes resultados corroboram os encontra- dos por Suehiro (2006) em sua avaliação com ADAPE de estudantes com escolaridade semelhante ao dos investigados no presente estudo. .Isto posto, verificou-se o desempenho em escrita comparativo entre as séries, tendo-se como critérios o desempenho no geral e diferenciando-o por nível de complexidade das palavras (Tabela 4). 
TABELA 4

Desempenho Médio e Índices Estatísticos do t de Student por Série

\begin{tabular}{ccccccc}
\hline Classificação & $\begin{array}{c}\mathbf{N} \\
\text { (palavras) }\end{array}$ & Série & $\begin{array}{c}\text { Média } \\
\text { ponderada }\end{array}$ & $\begin{array}{c}\text { Desvio } \\
\text { Padrão }\end{array}$ & $\boldsymbol{t}$ & $\boldsymbol{p}$ \\
Acertos & & 2 & 70,58 & 5,43 & $-7,83$ & 0,000 \\
& 114 & 3 & 88,61 & 5,02 & & \\
\hline \multirow{2}{*}{ Palavras sem dificuldades } & & 2 & 39,49 & 14,19 & & \\
& 54 & 3 & 46,68 & 9,08 & $-7,06$ & 0,000 \\
\hline \multirow{2}{*}{ Palavras com dificuldades } & & 2 & 21,94 & 12,16 & & \\
& 60 & 3 & 29,89 & 10,48 & $-7,94$ & 0,000 \\
\hline
\end{tabular}

Nota. $a \leq 0,01$.

Considerados o desempenho no ADAPE, focalizados os acertos por série, constata-se que há diferenças significativas entre $2^{\mathrm{a}}$ e $3^{\mathrm{a}}$ séries, sendo o desempenho melhor para a série mais avançada. Tais resultados corroboram os estudos de Zorzi (1998) Pinheiro e Rothe-Neves (2001), França, Wolf, Mooje e Rotta (2004) e Meirelles e Correa (2005) que observaram o efeito da escolaridade no desenvolvimento da escrita, quando utilizaram como medida de avaliação o ditado.

Ao lado disso, considerando-se que Theodório (2004) afirma ser o domínio da escrita uma conquista na vida acadêmica da criança e fundamental na for- mação do sujeito escritor, os resultados obtidos pelos participantes da $3^{\mathrm{a}}$ série revelam que estes apresentam domínio das regras ortográficas de modo mais estabelecido do que os de $2^{\mathrm{a}}$ série. Pode-se supor, neste caso, que dada a aquisição desta habilidade, os estudantes a utilizarão com um recurso que poderá vir a favorecer seu rendimento acadêmico posterior.

Visando analisar o tipo de erro apresentado nessa etapa pelos estudantes, procedeu-se à análise pelas categorias de dificuldade. Os critérios adotados foram os utilizados para a organização do ADAPE (Tabela 5).

TABELA 5

Média de Acertos por Tipo de Dificuldades das Palavras e Índices Estatísticos do t de Student por Série

\begin{tabular}{ccccccc}
\hline Categorias & $\begin{array}{c}\mathbf{N} \\
\text { (palavras) }\end{array}$ & Série & $\begin{array}{c}\text { Média } \\
\text { ponderada }\end{array}$ & Desvio Padrão & $t$ & $p$ \\
\hline Encontro consonantal & 17 & 2 & 9,13 & 5,43 & $-7,81$ & 0,000 \\
Dígrafo & 12 & 2 & 9,66 & 5,46 & $-7,32$ & 0,000 \\
& & 3 & 12,88 & 5,01 & & \\
Sílaba composta & 06 & 2 & 3,09 & 1,80 & $-7,20$ & 0,000 \\
& & 3 & 4,09 & 1,36 & & \\
Sílaba complexa & \multirow{2}{*}{13} & 2 & 7,22 & 3,77 & $-7,99$ & 0,000 \\
& & 3 & 9,81 & 3,36 & & \\
\hline
\end{tabular}

Ratifica-se a influência da escolaridade no processo de aquisição da escrita ortográfica quando se analisa o desempenho dos participantes em função da complexidade dos itens (Tabela 5). A categoria na qual foi verificada maior dificuldade foi a das palavras com sílabas compostas, enquanto àquelas com encontros consonantais e dígrafos foram as que os estudan- tes mais acertaram principalmente os de $3^{\mathrm{a}} \mathrm{s}$ séries. Em se constatando tais diferenças, a análise Multivariada de Médias (MANOVA) foi utilizada para verificar a existência de diferenças significativas entre a idade, o tipo de escola frequentada e o gênero por série para cada categoria de item (Tabelas 6 e 7). 
TABELA 6

Análise Multivariada de Médias (MANOVA) por Categoria de Item em Função do Tipo de Escola Frequentada, Gênero e Idade Para a $2^{\text {a }}$ Série

\begin{tabular}{|c|c|c|c|c|}
\hline Variável & Categoria de item & $g l$ & $F$ & $p$ \\
\hline \multirow{7}{*}{ Escola } & acerto & 3 & 5,70 & $0,01^{*}$ \\
\hline & consonantal & 3 & 6,95 & $0,00^{*}$ \\
\hline & dígrafo & 3 & 5,68 & $0,00^{*}$ \\
\hline & composta & 3 & 2,99 & $0,03^{*}$ \\
\hline & complexa & 3 & 4,73 & $0,00^{*}$ \\
\hline & simples & 3 & 4,99 & $0,00^{*}$ \\
\hline & dificuldades & 3 & 6,18 & $0,00^{*}$ \\
\hline \multirow{7}{*}{ Gênero } & acerto & 1 & 5,56 & $0,02^{*}$ \\
\hline & consonantal & 1 & 4,07 & $0,05^{*}$ \\
\hline & dígrafo & 1 & 6,63 & $0,01^{*}$ \\
\hline & composta & 1 & 3,26 & 0,07 \\
\hline & complexa & 1 & 2,87 & 0,09 \\
\hline & simples & 1 & 5,26 & $0,02^{*}$ \\
\hline & dificuldades & 1 & 5,45 & $0,02^{*}$ \\
\hline \multirow{7}{*}{ Idade } & acerto & 4 & 1,09 & 0,36 \\
\hline & consonantal & 4 & 0,77 & 0,54 \\
\hline & dígrafo & 4 & 0,81 & 0,52 \\
\hline & composta & 4 & 0,62 & 0,65 \\
\hline & complexa & 4 & 0,99 & 0,42 \\
\hline & simples & 4 & 1,72 & 0,15 \\
\hline & dificuldades & 4 & 0,70 & 0,59 \\
\hline
\end{tabular}

Nota. *significativo a $p<0,05$;

$g l=$ graus de liberdade;

F e $p=$ índices estatísticos.

Para os estudantes de $2^{\mathrm{a}}$ série, identificou-se diferença para todas as categorias de item para o tipo de escola frequentada. Os estudantes de escola particular revelaram melhor desempenho que os da escola pública. Quanto ao gênero, apenas não houve diferença significativa de desempenho entre meninas e meninos para os itens com sílaba composta e complexa. Para os demais tipos de itens, as meninas erraram menos que os meninos. Não se observou diferença por idade (Tabela 6). 


\section{TABELA 7}

Análise Multivariada de Médias (MANOVA) por Categoria de Item em Função do Tipo de Escola Frequentada, Gênero e Idade Para a $3^{\text {a }}$ Série

\begin{tabular}{|c|c|c|c|c|}
\hline Variável & Categoria de item & $g l$ & $F$ & $p$ \\
\hline \multirow{7}{*}{ Escola } & acerto & 3 & 3,80 & $0,01^{*}$ \\
\hline & consonantal & 3 & 5,16 & $0,00^{*}$ \\
\hline & dígrafo & 3 & 3,21 & $0,03^{*}$ \\
\hline & composta & 3 & 1,62 & 0,17 \\
\hline & complexa & 3 & 4,21 & $0,01^{*}$ \\
\hline & simples & 3 & 2,80 & $0,04^{*}$ \\
\hline & dificuldades & 3 & 4,14 & $0,01^{*}$ \\
\hline \multirow{7}{*}{ Gênero } & acerto & 1 & 0,30 & 0,59 \\
\hline & consonantal & 1 & 0,02 & 0,88 \\
\hline & dígrafo & 1 & 0,40 & 0,53 \\
\hline & composta & 1 & 0,39 & 0,54 \\
\hline & complexa & 1 & 0,36 & 0,55 \\
\hline & simples & 1 & 0,51 & 0,48 \\
\hline & dificuldades & 1 & 0,19 & 0,66 \\
\hline \multirow{7}{*}{ Idade } & acerto & 3 & 3,37 & $0,02^{*}$ \\
\hline & consonantal & 3 & 3,60 & $0,02^{*}$ \\
\hline & dígrafo & 3 & 3,83 & $0,01^{*}$ \\
\hline & composta & 3 & 2,21 & 0,09 \\
\hline & complexa & 3 & 2,47 & 0,07 \\
\hline & simples & 3 & 3,15 & $0,03^{*}$ \\
\hline & dificuldades & 3 & 3,82 & $0,01^{*}$ \\
\hline
\end{tabular}

Nota. *significativo a $p<0,05$;

$g l=$ graus de liberdade;

Fe $p=$ índices estatísticos.

Os estudantes de $3^{\mathrm{a}}$ série da escola particular apresentaram menos dificuldades na escrita que os da escola pública para todas as categorias de itens, exceção feita aos itens com sílabas compostas. Constatou-se também uma evolução da aquisição da escrita depen- dente do desenvolvimento, pois os alunos mais novos revelaram ter mais erros que os mais velhos. Tal diferença não foi observada para itens com sílabas complexas e composta. Não foi constatado desempenho diferenciado por gênero (Tabela 7). 


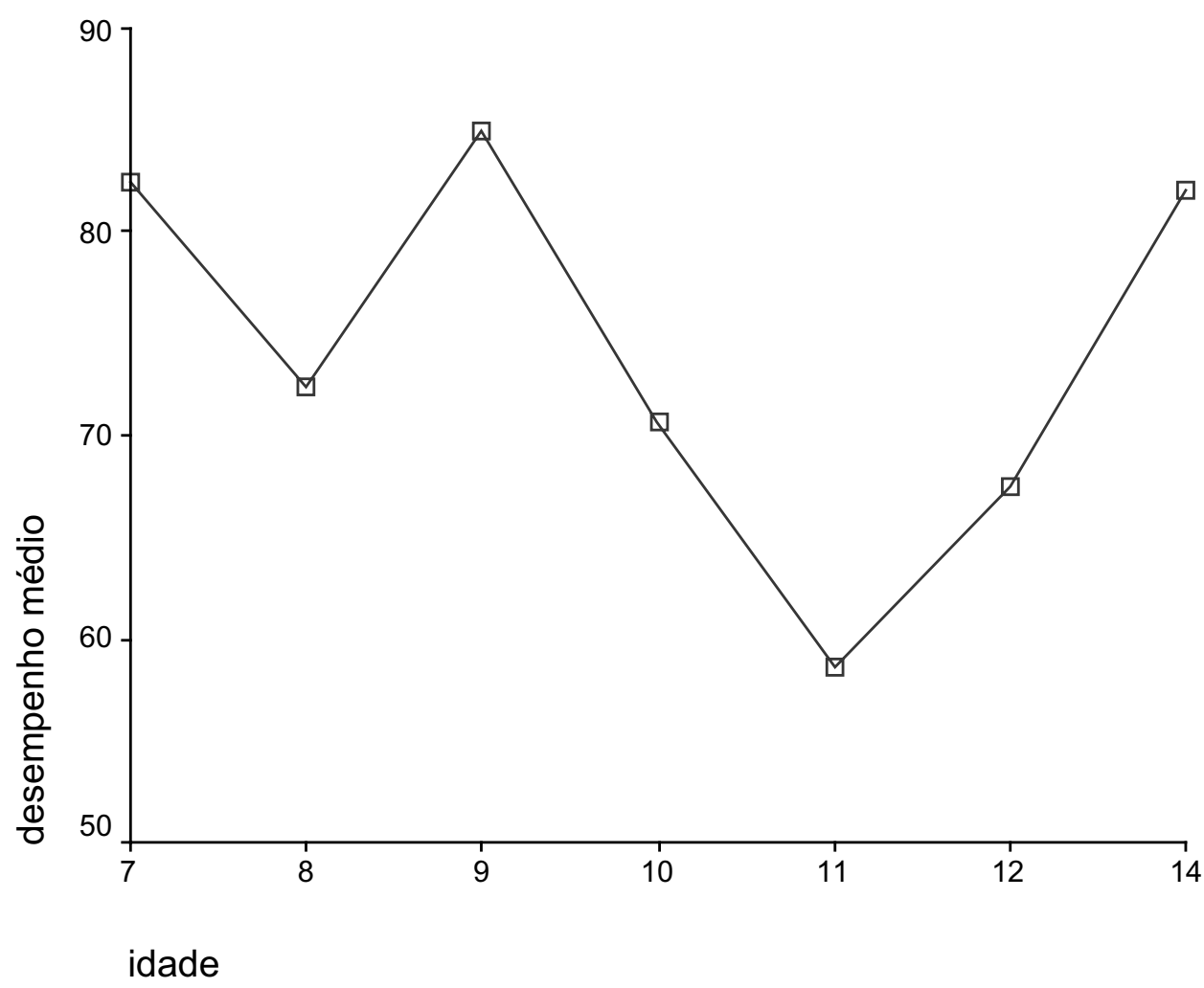

Figura 1. Desempenho médio dos participantes quanto à idade.

A análise do desempenho por idade mostrou que os alunos com faixa etária de 9 anos possuem um melhor desempenho acadêmico $(\mathrm{M}=84,93)$, em relação aos alunos de 11 anos $(M=58,56)$. Os alunos mais velhos, com 11 anos, apresentam defasagem idade/série, e dificuldade de aprendizagem na escrita (Figura 1). Estes dados reafirmam o desempenho superior dos participantes da $3^{\mathrm{a}}$ série neste estudo e corroboram o estudo de Pacheco e Sisto (2005).
A análise de variância do desempenho por idade por meio da ANOVA, independente da série, revelou que há diferenças significativas entre as idades dos participantes $(\mathrm{F}=5,283 ; p=0,000)$. O melhor desempenho também foi identificado para 9 anos $(\mathrm{M}=84,93$; $\mathrm{DP}=24,64)$ e o pior para 11 anos $(\mathrm{M}=58,56$; $\mathrm{DP}=$ 41,68). Resultados semelhantes foram encontrados por Pacheco e Sisto (2005) e Suehiro (2006).

TABELA 8

Desempenho dos Participantes por Gênero, Séries e Tipo de Escola Considerando-se os Índices do Teste $t$ de Student

\begin{tabular}{ccccccc}
\hline \multicolumn{2}{c}{ Variável } & N & Média & Desvio padrão & $\boldsymbol{t}$ & $\boldsymbol{p}$ \\
\hline \multirow{2}{*}{ gênero } & feminino & 278 & 83,45 & 25,02 & 5,09 & 0,000 \\
& masculino & 268 & 71,34 & 31,04 & & \\
\multirow{2}{*}{ série } & $2^{\text {a }}$ & 327 & 70,81 & 29,67 & $-6,93$ & 0,000 \\
& $3^{\text {a }}$ & 219 & 87,50 & 24,17 & & \\
\multirow{2}{*}{ escola } & pública & 497 & 76,03 & 29,31 & $-5,24$ & 0,000 \\
& particular & 49 & 92,47 & 16,26 & & \\
\hline
\end{tabular}

Nota. Diferenças significativas se $p \leq 0,05$. 
Há diferença significativa entre todas as variáveis analisadas (Tabela 8). Observa-se que as meninas possuem um melhor desempenho do que os meninos. No que se refere à série, os alunos da $3^{\mathrm{a}}$ série são os que apresentaram melhor média de acertos e a escola particular diferiu da pública, apesar da maior parte dos participantes ser da pública. Verifica-se na Tabela 8 que os melhores resultados observados no ADAPE pelos participantes do estudo são as meninas que cursam a $3^{\text {a }}$ série em escolas particulares. $O$ estudo de Guimarães (2003) revelou resultado semelhante ao do presente estudo no que se refere à série. Estes resultados corroboram o estudo de Suehiro (2006).

\section{CONSIDERAÇÕES FINAIS}

Diversos motivos podem levar às dificuldades de aprendizagem daí a necessidade de diferenciar as crianças que possuem baixo rendimento escolar com dificuldade de aprendizagem, das que possuem baixo rendimento escolar por motivos específicos (Alfonso \& Mascolo, 2002; Center for Mental Health in Schools, 2002; Flanagam, Ortiz). Dessa forma, uma avaliação objetiva de características da aprendizagem, do contexto educacional e do próprio estudante é relevante tanto para identificar os aspectos deficitários ou que comprometem o processo, quanto para a elaboração de intervenções adequadas a suas necessidades específicas (Joly, 2004; Sisto, 2001; Suehiro, 2006).

É necessário analisar as avaliações, principalmente as de leitura e escrita, feitas nas escolas a fim de buscar indicadores que possibilitem melhorar o rendimento acadêmico dos alunos. Isto posto, cabe refletir também sobre a necessidade do ensino das regras de estruturação da língua para a aprendizagem da escrita, bem como, da consciência fonológica e do reconhecimento do alfabeto enquanto habilidades preditivas para a escrita (Berninger \& cols., 1998; Bernardino \& cols., 2006; Capovilla, 1999; Cardoso- Martins, 1995; 2005; França \& col., 2004; Maluf \& Barrera, 1997; Snow, 2002). A pouca atenção dada a estas habilidades pode vir a acarretar um baixo rendimento acadêmico geral, como focaliza Sanches (1998) e como constatado pelos resultados do SAEB (INEP, 2006).

Neste sentido e dado o restrito número de instrumentos validados para avaliar a aquisição da leitura, escrita e outras habilidades cognitivas relacionadas à alfabetização, que possam ser utilizados por pesquisadores, professores e psicólogos (Capovilla, 2003), sugerem-se pesquisas futuras sobre este tema que possam vir a contribuir com a qualidade do ensino e com o desenvolvimento de testes específicos para este fim. Cabe também o desenvolvimento de programas que favoreçam a alfabetização dos alunos do primeiro ciclo do ensino fundamental.

\section{REFERÊNCIAS}

Bartholomeu, D., Sisto, F. F., \& Rueda, F. J. M. (2005). Dificuldades de aprendizagem na escrita e características emocionais de crianças. Psicologia em Estudo, 11, 139-146.

Bernardino Júnior, J. A., Freitas, F. R., Souza, D. G., Maranhe, E. A., \& Bandini, H. H. M. (2006). Aquisição de leitura e escrita como resultado do ensino de habilidades de consciência fonológica. Revista Brasileira de Educação Especial, 12, 423-450.

Berninger, W. V., Vaughan, K., Abbott, D. R., Brooks, A., Abbott, S. P., Rogan, L., Reed E., \& Graham, S. (1998). Early intervention for spelling problems: Teaching functional spelling units of varying size with a multiple-connections framework. Journal of Educational Psychology, 90, 587- 605.

Capellini, A. S., Tonelotto, F. M. J., \& Ciasca, M. S. (2004). Medidas de desempenho escolar: Avaliação formal e opinião de professores. Estudos de Psicologia, 21, 79-80.

Capovilla, A. G. S. (1999). Leitura, escrita e consciência fonológica: Desenvolvimento intercorrelações e intervenções. Tese de doutorado não-publicada, Instituto de Psicologia, Universidade de São Paulo.

Capovillla, A. G. S., Gutschow, C. R. D., \& Capovilla, F. C. (2004). Cognitive skills that predict competencies in reading and spelling. Psicologia: Teoria e Prática, 6, 13-26.

Cardoso-Martins, C. (1995). Sensitivity to rhymes, syllables and phonemes in literacy acquisition in portuguese. Reading Research Quarterly, 30, 808-827.

Cardoso-Martins, C., \& Batista, A. C. E. (2005). O conhecimento do nome das letras e o desenvolvimento da escrita: Evidências de crianças falantes do português. Psicologia: Reflexão $e$ Crítica, 18, 330-336.

Center for Mental Health in Schools (2002). An introductory packet on learning problems and learning disabilities. Los Angeles: UCLA.

Cunha, C. A., Sisto, F. F., \& Machado, F. (2006). Dificuldade de aprendizagem na escrita e autoconceito num grupo de crianças. Avaliação Psicológica, 5, 153-157.

De Voss, D., Cushman, E., \& Grabill, J. (2005). The infrastructure of composing: The when of new-media writing. College Composition and Communication, 57, 14-44.

Flanagam, D. P., Ortiz, S. O., Alfonso, V. C., \& Mascolo, J. T. (2002). The achievement test desk reference (ATDR). Boston: Allyn and Bacon.

França, M. P., Wolff, C. L., Moojen, S., \& Rotta, N. T. (2004). Aquisição da linguagem oral: Relação e risco para a linguagem escrita. Arquivos de Neuropsiquiatria, 62(2-B), 469-472.

Goswami, U., \& Bryant, P. (1997). Phonological skills and learning to read. Hove, UK: Psychology Press.

Guimarães, K. R. S. (2003). O aperfeiçoamento da concepção alfabética de escrita: Relações entre consciência fonológica e representações ortográficas. Em M. R. Maluf (Org.), Metalinguagem e aquisição da escrita: Contribuições da pesquisa para a prática da alfabetização (pp. 149-179). São Paulo: Casa do Psicólogo. 
Instituto Nacional de Estudos e Pesquisas Educacionais Anísio Teixeira - INEP (2006). Qualidade da educação: Uma nova leitura do desempenho dos estudantes de $4^{a}$. série do ensino fundamental. Retirado em 16 de outubro de 2006, de http://www.inep.gov.br/download/saeb/2003/boletim_4serie.pdf.

Jaeger, A., Schossler, T., \& Wainer, R. (1998). Comparative study between the process of writing acquisition in children and adults. Psicologia: Reflexão e Critica, 11(3), 551-558.

Joly, M. C. R. A. (2004). Escrita criativa: Avaliando estratégias e procedimentos educacionais eficazes para o ensino fundamental. Em G. P. Witter (Org.), Psicologia e educação: Professor, ensino e aprendizagem (pp. 59-74). Campinas: Alínea.

Juel, C., Griffith, P. L., \& Gough, P. B. (1986). Acquisition of literacy: A longitudinal study of children in first and second grade. Journal of Educational Psychology, 78(4), 243-255.

Juzwik, M. M., Curcic, S., Wolbers, K., Moxley, K. D., Dimling, L. M., \& Shankland, R. K. (2006). Writing into 21st century: An overview of research on writing: 1999 to 2004. Written Communication, 23, 451-476.

Maluf, M. R., \& Barrera, S. D. (1997). Consciência fonológica e linguagem escrita em pré-escolares. Psicologia: Reflexão e Crítica, 10, 125-145.

Meirelles, E. S., \& Correa, J. (2005). Regras contextuais e morfossintáticas na aquisição da ortografia da língua portuguesa por crianças. Psicologia: Teoria e Pesquisa, 21(1), 77-84.

Ministério da Educação e Cultura (1997). Parâmetros Curriculares Nacionais: Introdução. Brasília: MEC.

Nunes, L. D. (2002). Avaliação de dificuldades de aprendizagem na escrita: Estudo comparativo do desempenho no ADAPE, em provas operatórias e de procedimento e em subtestes do WISC. Tese de doutorado não-publicada, Universidade Estadual de Campinas.

Pacheco, L., \& Sisto, F. F. (2005). Ajustamento social e dificuldade de aprendizagem. Psic: Revista de Psicologia da Vetor Editora, 6, 43-50.

Pinheiro, A. M., \& Rothe-Neves, R. (2001). Avaliação cognitiva de leitura e escrita: As tarefas de leitura em voz alta e ditado. Psicologia: Reflexão e Crítica, 14, 399-408.

Sánchez, J. N. G. (1998). História y concepto de las dificultades de aprendizaje. Em V. S. Bermejo \& J. B. Llera (Orgs.), Dificultades de aprendizaje (pp. 17-46). Espanha: Síntesis.
Sisto, F. F. (2001). Dificuldades de aprendizagem. Em F. F. Sisto, E. Boruchovitch, L. D. T. Fini, R. P. Brenelli \& S. de C. Martinelli (Orgs.), Dificuldades de aprendizagem no contexto psicopedagógico (pp. 19-35). Petrópolis: Vozes.

Sisto, F. F. (2001a). Dificuldades de aprendizagem em escrita: Um instrumento de avaliação (ADAPE). Em F. F. Sisto, E. Boruchovitch, L. D. T. Fini, R. P. Brenelli \& S. de C. Martinelli (Orgs.), Dificuldades de aprendizagem no contexto psicopedagógico (pp. 190-213). Petrópolis: Vozes.

Sisto, F. F. (2002). Avaliação de dificuldade de aprendizagem: Uma questão em aberto. Em F. F. Sisto, E. A. Dobránszky \& A. Monteiro (Orgs.), Cotidiano escolar: Questões de leitura, matemática e aprendizagem (pp. 121-136). Petrópolis: Vozes.

Sisto, F. F., \& Fernandes, D. C. (2004). Dificuldades linguísticas na aquisição da escrita e agressividade. Psicologia Escolar e Educacional, 8, 75-84.

Snow, C. (2002). Reading for understanding. Santa Monica: RAND.

Stanovich, K. E., Cunningham, A. E., \& Cramer, B. B. (1984). Assessing phonological awareness in kindergarten children: Issues of task comparability. Journal of Experimental Child Psychology, 38, 175-190.

Suehiro, A. C. B. (2006). Dificuldade de aprendizagem da escrita num grupo de crianças do ensino fundamental. Psic: Revista de Psicologia da Vetor Editora, 7, 59-68.

Suehiro, A. C. B., \& Santos, A. A. A. (2005). O Bender e as dificuldades de aprendizagem: Estudo de validade. Avaliação Psicológica, 4, 23-31.

Treiman, R., \& Baron, J. (1983). Phonemic-analysis training helps children benefit from spelling-sound rules. Memory \& Cognition, 11(4), 382-389.

Yopp, H. K. (1988). The validity and reliability of phonemic awareness tests. Reading Research Quarterly, 23, 159-177.

Zorzi, J. L. (1998). Aprender a escrever: A apropriação do sistema ortográfico. Porto Alegre: Artes Médicas.

Recebido: 20/11/2007

Última revisão: $14 / 02 / 2009$

Aceite final: 01/09/2009

\section{Sobre as autoras:}

Maria Cristina Rodrigues Azevedo Joly: Psicóloga; Doutora em Psicologia Educacional e do Desenvolvimento Humano pela Universidade de São Paulo e docente na Graduação e Pós-Graduação stricto sensu em Psicologia da Universidade São Francisco.

Débora Pereira de Barros: Psicóloga; Mestre em Avaliação Psicológica pela Universidade são Francisco.

Janete Aparecida da Silva Marini: Pedagoga; Mestre em Avaliação Psicológica pela Universidade São Francisco; Doutoranda em Educação pela Unicamp; docente na Graduação em Pedagogia do Centro Universitário Padre Anchieta; Diretora da Educação Infantil da Rede de Ensino Municipal de Jundiaí.

Endereço para correspondência: Maria Cristina Rodrigues Azevedo Joly - Rua Alexandre Rodrigues Barbosa, 45 - 13251-900 Itatiba/SP. Endereço eletrônico: cristina@trtec.com.br. 